\title{
Measuring the Process Parameters of the IBAD Method
}

\author{
M. Zoriy, F. Černý, D. Palamarchuk, S. Konvičková, I. Hüttel
}

Chromium nitride films are known as good protective layers for against both corrosion and wear. These coatings have been studied in detail during recent years. Their protective capability strongly depends on the deposition conditions. A modern method for preparing chromium nitride is the IBAD (Ion Beam Assisted Deposition) method.

The main parameter determining the composition and properties of the films prepared by the IBAD method is the arrival ratio of impinging nitrogen ions to chromium atoms.

In order to calibrate the ion beam XY-mechanical scanner with a Faraday cup, a detector was designed and constructed. By mathematical processing of the data, the flux of the nitrogen atoms was found. To obtain the flux of the chromium atoms the RBS and Talystep methods were used.

Now, on the basis of this data, we can perform $\mathrm{CrN}_{x}$ coatings with controlled composition and properties.

Keywords: IBAD, $\mathrm{CrN}, \mathrm{CrN}_{2}$, calibration, electron beam.

\section{Introduction}

In recent years the hard coatings have play a major role in mechanical engineering. Hard coatings are used to increase resistance against wear and fatigue.

Many kinds of hard coatings are now known. The most widely used is titanium nitride, because of its high hardness [1]. Chromium nitride is used for the same propose [2]. In addition to high hardness it has a very good corrosion resistance, especially under high temperatures in various atmospheres. The hardness and friction properties of chromium nitride coatings depend on the structure and orientation of the crystals in the film [3]. The corrosion properties also depend on the porosity/density of the coatings [4]. The importance of the deposition parameters is critical.

There are many methods for preparing these coatings, which can be classified in various ways. Effective modern methods are based on plasma and ion beam processing technology. One of these is the IBAD method [5].

A schematic view of the experimental arrangement for the IBAD method is shown in Fig 1.

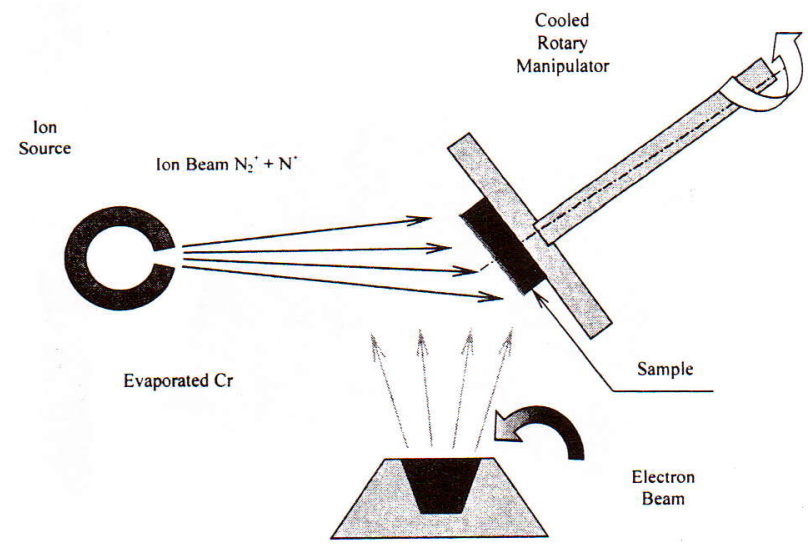

Fig. 1: Schematic view of experimental arrangement for the IBAD method
It involves deposition of a desired material using a vacuum electron beam evaporation technique and simultaneous bombardment by an energetic ion beam.

Chromium nitride has two stoichiometric modifications $\mathrm{CrN}$ and $\mathrm{Cr}_{2} \mathrm{~N}$ [6]. However, we will be preparing a customary composition $\mathrm{CrN}_{\mathrm{x}}$, where $x$ is in the range of $[0 \div 1]$, and we will investigate the characteristics of the process in dependence on the $x$ ratio. To do this, we need to calibrate the deposition subprocesses - the atomic fluxes of chromium and nitrogen in IBAD equipment.

\section{Calibration of an ion beam}

A flow of nitrogen of high purity is fed into the ion source, where ionization of the nitrogen takes place. The ion source was developed from the Franks Twin Anode design. It consists of a graphite outer tube with closed ends, mounted in an insulator. Inside the tube there are two tungsten electrodes, closely spaced about the centerline of the tube.

At a distance of $35 \mathrm{~mm}$ away, there is a corresponding wider slit in the extractor electrode. During operation a high tension of $90 \mathrm{keV}$ is supplied to the ion source.

The large potential difference between the positive HT in the ion source and the zero voltage of the extractor electrode provides the force to accelerate the positive nitrogen ions to a high velocity and form the ion beam. Once the ions pass through the slit in the extraction electrode they continue at constant velocity until they hit the work piece. The ion beam is a mixture of molecular and atomic ions in proportion 3:1.

As a goal we need to know the quantity of nitrogen atoms that the sample per unit area per unit time - the flux of the nitrogen atoms. The TECVAC 221 Ion Implanter has no suitable measuring instrument for this, so we made an appropriate device ourselves and used it to perform the calibration of the ion beam. 
This device is a mechanical XY-scanner that consists of a Faraday cup detector, two stepping-motors, a scrolling mechanism and other accessories.

A program was written in $\mathrm{C}$ language to control two stepping-motors, which moved the detector into selected points of cross-sections of the ion beam. An XY-scanner was applied in the region of dimensions $200 \times 200 \mathrm{~mm}$ with a step of $2 \mathrm{~mm}$. Another program estimated the value of ion beam density and plotted the graph that illustrated the profile of ion beam density. The resulting distribution of ion beam density in the TECVAC Ion Implanter is shown in Fig. 2. This is the profile of the ion beam current density, but we need a profile of the flux of the nitrogen atoms. We therefore need to perform a mathematical procedure to transform ion beam density into atomic nitrogen flux. We calculated the quantity of nitrogen atoms as follows:

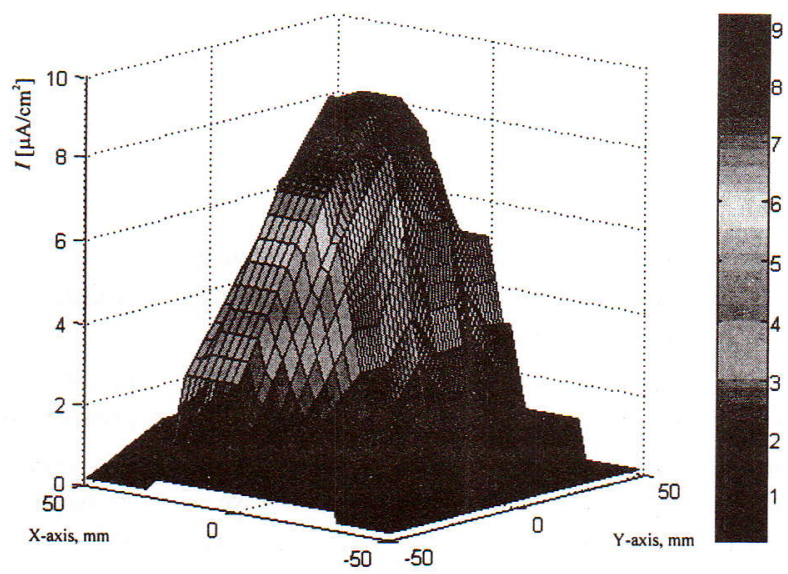

Fig. 2: Profile monitoring of total flux of ions

\section{Given data:}

$I=9.2 \mu \mathrm{A} / \mathrm{cm}^{2}-$ example of value of ion beam density (measured by Faraday cup detector);

$\mathrm{e}=1.6021892 \cdot 10^{-19} \mathrm{C}$ - electron charge magnitude;

$P_{N}$ - flux of atomic nitrogen ions;

$P_{2 N}$ - flux of molecular nitrogen ions;

$P$ - total flux of ions;

$N_{N}$-flux of nitrogen atoms included in atomic nitrogen ions;

$N_{2 N}$ - flux of nitrogen atoms included in molecular nitrogen ions;

$N$ - total flux of nitrogen atoms.

\section{Procedure for Solution:}

We know the ion beam density and charge of one ion. From this data we are able to calculate the total flux of ions: $P=\frac{1}{e}=\frac{0.0000092}{1.60221892 \cdot 10^{-19}}=5.742 \cdot 10^{13} \mathrm{~cm}^{-2} \cdot \mathrm{s}^{-1}-$ total flux of ions;

$\frac{P_{2 N}}{P_{N}}=\frac{3}{1}-$ number of molecular ions: number of atomic ions;

$P=P_{N}+P_{2 N} \Rightarrow \frac{P}{P_{N}}=1+3=4$.
So the fluxes of the atomic and molecular ions will be, respectively:

$P_{N}=\frac{P}{4}=\frac{5.742 \cdot 10^{13}}{4}=1.4355 \cdot 10^{13} \mathrm{~cm}^{-2} \cdot \mathrm{s}^{-1}$;

$P_{2 N}=P-P_{N}=5.742 \cdot 10^{13}-1.4355 \cdot 10^{13}=$

$=4.3065 \cdot 10^{13} \mathrm{~cm}^{-2} \cdot \mathrm{s}^{-1}$

The flux of the atoms included in the atomic nitrogen ions equals the flux of the nitrogen ions:

$$
\mathrm{N}_{\mathrm{N}}=\mathrm{P}_{\mathrm{N}}=1.4355 \cdot 10^{13} \mathrm{~cm}^{-2} \cdot \mathrm{s}^{-1} \text {; }
$$

The flux of the atoms included in the molecular nitrogen ions equals flux of equal twice the flux of the molecular nitrogen ions:

$\mathrm{N}_{2 \mathrm{~N}}=2 \cdot \mathrm{P}_{2 \mathrm{~N}}=8.613 \cdot 10^{13} \mathrm{~cm}^{-2} \cdot \mathrm{s}^{-1}$

So the total flux of nitrogen atoms is:

$$
N=N_{N}+N_{2 N}=1.005 \cdot 10^{14} \mathrm{~cm}^{-2} \cdot \mathrm{s}^{-1}
$$

- total flux of nitrogen atoms.

The Profile monitoring of the flux of atoms is shown in Fig. 3

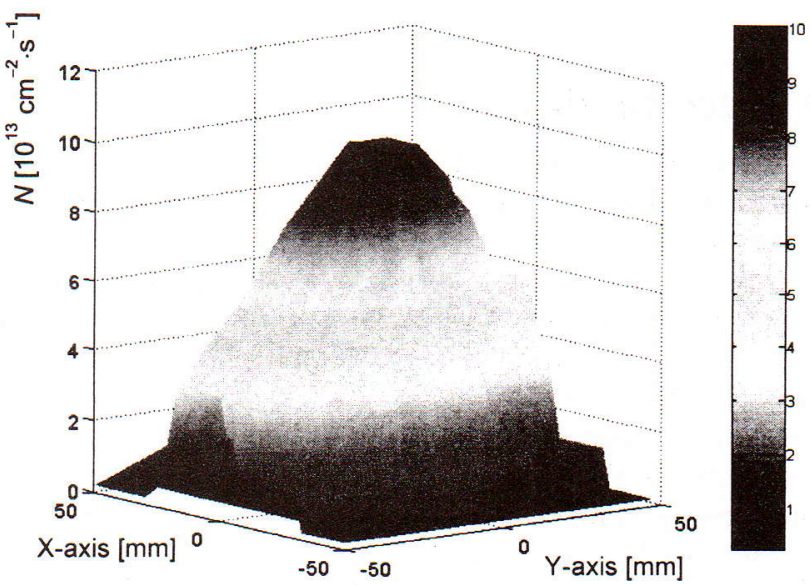

Fig. 3: Profile monitoring of total flux of nitrogen atoms

Another view of the same profile is presented in Fig. 4 . This figure shows that the profile of the flux has its maximum in the ellipse region, extended along the $\mathrm{X}$-axis.

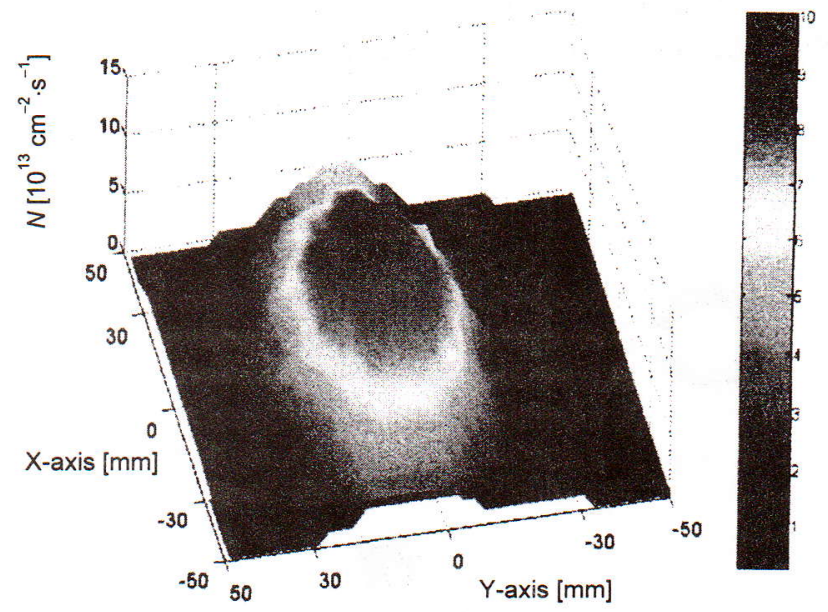

Fig. 4: Profile monitoring of total flux of nitrogen atoms (another view) 
Fig. 5 shows a view from below of the profile monitoring of the total flux of the nitrogen atoms, and gives more information about the distribution the flux in the cross-section of the ion beam. Using this figure we can select a zone approximately $50 \mathrm{~mm}$ long and $30 \mathrm{~mm}$ wide, where the flux of the nitrogen will vary within a range of $15 \%$.
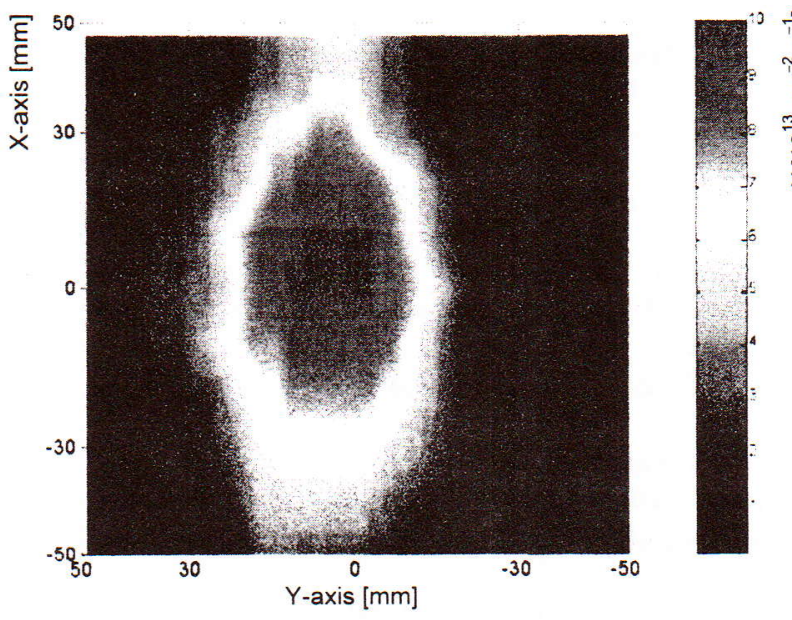

Fig. 5: Profile monitoring of total flux of nitrogen atoms, seen from below

So if we locate our samples in this region we will achieve satisfactory homogeneity and will be able to control the quantity of the nitrogen atoms that arrive on a unit area per one second.

\section{Calibration of the electron beam evaporation system}

To check the composition of chromium nitride we also need to calibrate the flux of the evaporated chromium atoms.

In the TECVAC Ion Implanter the evaporation is supplied by an electron beam gun. When the electron beam gun is running, it produces a powerful beam of electrons that are accelerated by negative high-tension up to $-10 \mathrm{kV}$. This electron beam melts and evaporates the chromium that deposits on the sample.

Besides depositing chromium on the sample that is mounted on the rotary manipulator, chromium is also deposited on the quartz crystal microbalance. This crystal oscillates with an original frequency, of $5 \mathrm{MHz}$, and the frequency of oscillation decreases with the mass of deposited chromium. In this way we can measure the thickness of the deposited coatings.

To check the measured thickness of the coatings, the Talystep method was used. In this method, a thin needle moves along the surface and detects the step between the surface of the substrate and the surface of the coating. Then the value of this step is converted into an electrical signal and the thickness of the coating is displayed.

To obtain the quantity of the chromium atoms in our coatings, the RBS (Rutherford Backscattering Spectroscopy) method was used. Our samples were bombarded by alpha particles with energy $2 \mathrm{MeV}$. By measuring the energy of the backscattered particles the exact quantity of chromium atoms was determined. Examples of the energetic spectra are shown in Fig. 6 and Fig. 7.

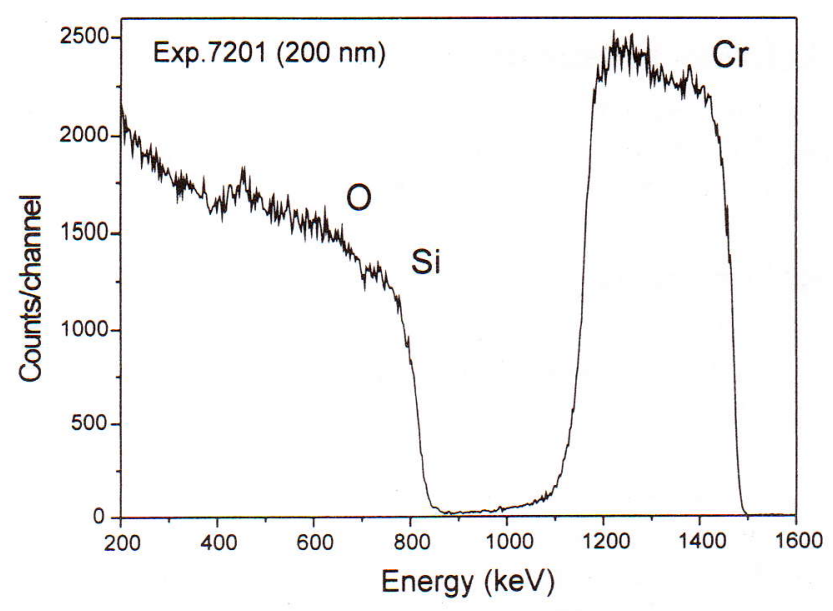

Fig. 6: Sample $200 \mathrm{~nm}, 2 \mathrm{MeV}$ alpha particles. The quantity of $\mathrm{Cr}$ atoms is $2.1 \cdot 10^{15} \mathrm{~cm}^{-2}$.

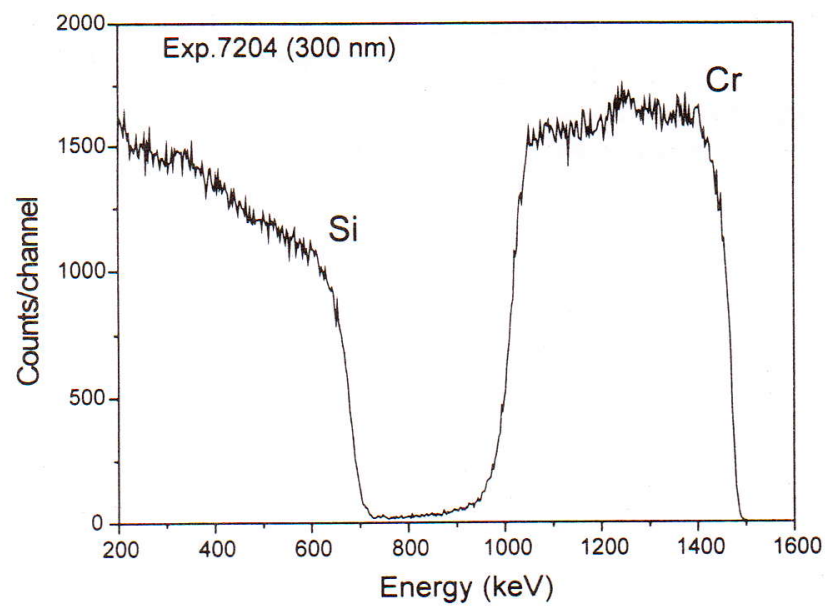

Fig. 7: Sample $300 \mathrm{~nm}, 2 \mathrm{MeV}$ alpha particles. The quantity of $\mathrm{Cr}$ atoms is $5.05 \cdot 10^{15} \mathrm{~cm}^{-2}$.

By comparing this data with the data measured by our IBAD equipment we calibrated the electron beam evaporation system.

For example, when the electron beam current was $100 \mathrm{~mA}$, the flux of the chromium atoms was $2 \cdot 10^{13} \mathrm{at} \cdot \mathrm{cm}^{-2}$.

\section{Conclusions}

Our ultimate goal is to prepare coatings of chromium nitride. To check the properties of these coatings we need to calibrate the deposition subprocesses - the atomic fluxes of chromium and nitrogen in the IBAD equipment. These calibrations have been done.

In order to calibrate the ion beam we designed and constructed an XY-mechanical scanner with a Faraday cup detector. By mathematical processing of the data we found the flux of the nitrogen atoms.

To obtain the flux of the chromium atoms we used the RBS and Talystep methods. 
Now, on the basis of this data, we can perform $\mathrm{CrN}_{\mathrm{x}}$ coatings with controlled composition and properties.

\section{Acknowledment}

This research has been supported by GACR grant No. 106/02/1194 and conducted within the framework of research project No. J04/98:210000012.

\section{References}

[1] Galvanetto, E., Galliano, F. P., Fossati, A., Borgioli, F.: Surf. Coat. Technol., Vol. 44, 2002, p. 1593-1606.

[2] Mernagh, V. A., Kelly, T. C., Ahern, M., Kennedy, A. D., Adriansen, A. P. M., Ramackers, P. P. J., McDonnel, L., Kockoek, R.: Surf. Coat. Technol., Vol. 49, 1991, p. 462.

[3] Smidt, F. A.: Int. Mater. Rev., Vol. 35, 1990, p. 61.

[4] Kumar, S., Rajul, V. S., Arunachalam, J., Khanna, A., Prasad, K.: Thin Solid Films, Vol. 388, 2001, p. 195-200.

[5] Pakala, M., Lin, R. Y.: Surf. Coat. Technol., Vol. 81, 1996, p. 233-239.

[6] Cunha, L., Andritschky, M., Rebouta, L., Pischow, K.: Surf. Coat. Technol., Vol. 119, 1999, p. 1152-1160.

Ing. Miroslav Zoriy

e-mail: mzorij@seznam.cz
Doc. Ing. František Černý, CSc. phone: +420224352437 e-mail: cernyf@fsid.cvut.cz

Ing. Dmytro Palamarchuk

Department of Physics

Doc. Ing. Svatava Konvičková, CSc. phone: +420224352511 e-mail:konvicko@fsid.cvut.cz

Department of Mechanics

Czech Technical University in Prague Faculty of Mechanical Engineering Technická 4,

16607 Prague 6, Czech Republic

Doc. Ing. Ivan Hüttel, CSc.

phone: +420224353205

e-mail: huttel@vscht.cz

Institute of Chemical Technology

Technická 3,

16628 Prague 6, Czech Republic 